\title{
Observation of Helical Edge Transport in a Screened Graphene
}

\author{
Helical quantum Hall phase in graphene on $\mathrm{SrTiO}_{3}$ \\ Authors: Louis Veyrat, Corentin Dèprez, Alexis Coissard, Xiaoxi Li, Frédéric Gay, \\ Kenji Watanabe, Takashi Taniguchi, Zheng Han, Benjamin A. Piot, Hermann Sellier, \\ and Benjamin Sacépé \\ arXiv:1907.02299

\begin{abstract}
Recommended with a Commentary by Leonid I. Glazman, Yale University
\end{abstract}

The strength of electron-electron interaction in a conductor is conventionally characterized by parameter $r_{s}=e^{2} /\left(\epsilon \hbar v_{F}\right)$. This dimensionless parameter is the ratio of the Coulomb interaction energy between two electrons separated by a typical distance, i.e., by the Fermi wavelength, to the Fermi energy in a gas of free fermions $\left(v_{F}\right.$ is the Fermi velocity and $\epsilon$ is the background dielectric constant of the medium). Starting from a Fermi gas with a non-degenerate ground state, the system would continuously "morph" into a Fermi liquid upon increase of the interaction from $r_{s}=0$ to some moderate value. The Fermi liquid description works perfectly well, say, for alkali metals having $r_{s} \sim 1$, with the ground state and quasiparticle excitations being direct descendants of those for the free fermions.

Situation changes drastically if the free-fermion ground state is degenerate: introduction of interactions then may result in emergence of new electron phases. The free-fermion spectrum may be "flattened" by applying even a small out-of-plane magnetic field to a twodimensional gas of free fermions. Landau quantization gives rise to discrete single-particle energy levels which are flat in the bulk and bend at the boundaries, creating states propagating along the boundary. Electron-electron interaction in a partially-occupied Landau level may result in a variety of phases, including the well-studied phases of the fractional quantum Hall effect.

The plot thickens if the free fermions are "living" in a graphene lattice and, in addition, their Fermi level is tuned to the charge neutrality point. Application of a small out-ofplane magnetic field creates a flat level half-filled with electrons carrying the spin and valley labels. In the absence of Zeeman splitting, a slight shift of Fermi level up in energy would create a particle-like state propagating along the edge, while a down-shift would lead to a state propagating in the opposite direction. Ignoring Zeeman energy and interactions, electron states at the charge-neutrality point carry a four-fold degeneracy, on top of the degeneracy associated with the Landau level. How interaction would affect such highlydegenerate system? 
Theory envisioned a variety of options which include an ordering which breaks the valley symmetry, or spin symmetry, or creates an order engaging both valley and spin indices of electrons $[1,2,3]$; the somewhat depressing (albeit unsurprising) conclusion was that the winning order is defined by the microscopic details of the interaction [4].

The least exciting option is a valley order leading to a version of a Mott insulator with a typical activation dependence of electric conduction at low temperatures. The most intriguing one is a spontaneously spin-polarized phase in which electron states with, say, spin-up are fully occupied while flipping a spin costs a finite energy. The spin-polarized phase is distinguished [5] by the appearance of an edge mode in which electrons with opposite spins propagate in opposite directions along the same boundary (aka helical edge modes). Due to the edge modes, the system may remain conducting at low temperatures. Scattering between the counter-propagating electron states within the helical mode renders conductance non-quantized. However, in the absence of spin-orbit coupling, electron motion in the helical mode is protected from elastic backscattering by conservation of the spin projection on the polarization axis, so the conductance should increase with lowering the temperature, approaching the quantized value of $e^{2} / h$ per edge.

A graphene sheet left to its own devices prefers an insulating phase (possibly a canted antiferromagnetic state), but experiments [6] did show that the system may be "coerced" into the spin-polarized phase by applying a sufficiently strong in-plane magnetic field, so that the total field is about 30T. It means that the energy difference between the two phases is a few meV's per electron, a fairly small fraction of a naïve estimate of $\sim 1 \mathrm{eV}$ based on the Coulomb potential at an inter-electron distance. The smallness of energy difference brings a hope that by tweaking interactions just a little one may nudge the system into a spontaneous formation of the spin-polarized state. The authors of Ref. [7] discovered that dielectric screening does the trick.

In the experiment [7], the graphene sheet was separated by mere $2-5 \mathrm{~nm}$ of a HBN spacer from $\mathrm{SrTiO}_{3}$ substrate. The latter has a huge $\epsilon \sim 10^{4}$ and may provide the dielectric screening for the electron-electron interaction in graphene. The effectiveness of screening does depend on the spatial structure of electron states. In a magnetic field, those may be thought of as pancakes of radius defined by magnetic length, $l_{B}=\sqrt{\hbar / e B}$. The larger $l_{B}$ is, the more effective is the screening. The inter-electron interaction is reduced by a factor of 10 at $l_{B} \approx 26 \mathrm{~nm}$ corresponding to field $B=1 \mathrm{~T}$. Transport measurements performed at that value of out-of-plane field (and at zero in-plane component!) produced a convincing evidence for the helical edge states.

To demonstrate the presence of the edge states and their helical nature, the authors performed a battery of well-established [8] tests involving the measurements of two- and multi-terminal non-local conductance. These states were observed in fields as small as $1 \mathrm{~T}$ and persisted till $B \approx 5 \mathrm{~T}$. At higher fields, a gap in the excitations spectrum apparently opens and grows (approximately linearly) with $B$; it results in an activation temperature dependence of the conductance. Opening of the gap at these stronger fields is consistent with the decreased effectiveness of the dielectric screening for pancakes of a smaller radius $l_{B}$.

Measurements attest to a certain robustness of the helical edge conductance which remains approximately quantized for channels as long as $1 \mu \mathrm{m}$ and for temperatures $T$ as high as $100 \mathrm{~K}$. The data (see Figs. 4A and $4 \mathrm{~B}$ of Ref. [7]) actually suggest that the most accurate 
quantization happens at $T=90 \mathrm{~K}$, rather than at $T=5 \mathrm{~K}$. That, along with the negative (albeit fairly small) temperature coefficient of the resistance $d R / d T$ may warrant further studies. The evident from Fig. $4 \mathrm{C}$ reentrant (in temperature) behavior of the ferromagnetic transition [9] presents even a bigger puzzle.

\section{References}

[1] K. Yang, S. Das Sarma, and A. H. MacDonald, Phys. Rev. B 74, 075423 (2006).

[2] K. Nomura and A. H. MacDonald, Phys. Rev. Lett. 96, 256602 (2006).

[3] H. A. Fertig and L. Brey, Phys. Rev. Lett. 97, 116805 (2006).

[4] M. Kharitonov, Phys. Rev. B 85, 155439 (2012).

[5] D. A. Abanin, P. A. Lee, and L. S. Levitov, Phys. Rev. Lett. 96, 176803 (2006).

[6] A. F. Young, J. D. Sanchez-Yamagishi, B. Hunt, S. H. Choi, K. Watanabe, T. Taniguchi, R. C. Ashoori, and P. Jarillo-Herrero, Nature 505, 528-532 (2014).

[7] Louis Veyrat, Corentin Dèprez, Alexis Coissard, Xiaoxi Li, Frédéric Gay, Kenji Watanabe, Takashi Taniguchi, Zheng Han, Benjamin A. Piot, Hermann Sellier, and Benjamin Sacépé, arXiv:1907.02299

[8] Markus König, SteffenWiedmann, Christoph Brüne, Andreas Roth, Hartmut Buhmann, Laurens W Molenkamp, Xiao-Liang Qi, and Shou-Cheng Zhang, Science 318, 766-770 (2007).

[9] I thank Herb Fertig for pointing out this feature to me. 